\title{
Drought Stress-induced Physiological and Metabolic Changes in Leaves of Two Oil Tea Cultivars
}

\author{
Xinjing Qu, Hui Wang, Ming Chen, Jiao Liao, and Jun Yuan \\ Key Laboratory of Cultivation and Protection for Non-Wood Forest Trees, Ministry of Education, \\ Central South University of Forestry and Technology, Changsha, Hunan 410004, China \\ Genhua Niu \\ Texas A\&M AgriLife Research and Extension Center at Dallas, Texas A\&M University, 17360 Coit \\ Road, Dallas, TX 75252
}

\begin{abstract}
AdDITIONAl INDEX wORDs. amino acids, Camellia oleifera, carbohydrates metabolites, organic acids, photosynthesis
Abstract. Oil tea (Camellia oleifera) is an important edible oil tree. However, its growth and yield are strongly limited by drought. This study investigated the physiological and metabolic responses of two common oil tea cultivars, Huajin and Changlin53, to moderate and severe drought stress. Based on the photosynthetic and physiological indices, 'Changlin 53' may be more tolerant to drought than 'Huajin'. A total of 41 key metabolites induced by drought stress, including 12 amino acids, 12 organic acids, 10 carbohydrates, 3 fatty acids, and 4 phenols, have been identified by liquid chromatography-mass spectrometry. Under moderate drought stress, the contents of carbohydrates, amino acids, and some organic acids in 'Changlin53' were significantly increased; however, under severe drought stress, the contents of soluble sugars were decreased and the synthesis ability of amino acids and organic acids were enhanced. The glutamic acid-mediated proline biosynthesis pathway and salicylic acid synthesis were continuously upregulated in 'Changlin53' under moderate and severe drought stress, which could regulate osmotic pressure and maintain intracellular environmental stability. Under moderate drought stress, the contents of monosaccharides, amino acids, and organic acids increased in 'Huajin' leaves. Furthermore, the shikimic acid-mediated secondary metabolite synthesis pathway was weakened. More secondary metabolites were used to increase glycolysis and tricarboxylic acid cycle to accelerate energy production and to enhance the glutamic acid-mediated proline biosynthesis pathway, which are necessary to increase osmotic regulation. Under severe drought stress, the contents of carbohydrates, organic acids, and some amino acids were significantly decreased in 'Huajin' leaves, indicating serious damage. These results deepened our understanding of the mechanisms involved in oil tea drought tolerance, which will help improve water management of oil tea seedlings.
\end{abstract}

Oil tea, an important edible oil tree species of Camellia, along with olive (Olea europaea), oil palm (Elaeis guineensis), and coconut (Cocos nucifera), are the four major woody oil species (Yang et al., 2016). Oil tea has been cultivated for more than 2300 years in southern China (Zhuang, 2008). It is known as "eastern olive oil" because of its high content of unsaturated fatty acids, similar to olive oil (Dong et al., 2017; Yang et al., 2016). At present, there is an urgent need to increase the selfsufficiency of edible oil in China (Dong et al., 2017). Oil tea has become a widely cultivated oil tree species in southern China because of its tolerance to barren soil and its long fruit production period ( $>80$ years) (Yang et al., 2016). The planting area of oil tea has reached 4 million hectares, which is widely distributed in 14 provinces in southern China, mostly in Hunan, Guangxi, and Jiangxi (Li et al., 2012).

Drought stress is one of the main environmental factors affecting plant survival and crop yield (Kudo et al., 2017). The effects of drought stress on plants depend on the intensity and duration of drought stress and the tolerance of cultivars (Marcek et al., 2019). The stomatal conductance $\left(g_{\mathrm{S}}\right)$ of leaves

Received for publication 17 June 2019. Accepted for publication 3 Sept. 2019. We thank the Provincial Key R\&D Program of Hunan, China (2017NK2201) for financial support.

J.Y. and G.N. are corresponding authors. E-mail: yuanjunchina@126.com or gniu@ag.tamu.edu. usually decreased at the beginning of drought stress, which limited the uptake of $\mathrm{CO}_{2}$ and decreased the photosynthetic rate (Drapal et al., 2017; Kebbas et al., 2015). However, longterm severe drought stress (SDS) not only affected photosynthetic rate but also decreased chlorophyll content and caused cell membrane lipids peroxide and cell structure destruction (Jaleel et al., 2009; Liang et al., 2019). Oil tea is mostly planted in low mountains and hilly areas. In these areas, soil moisture is lost faster than on flat land, and irrigation is difficult. High temperatures and drought in summer in southern China negatively affect the growth of oil tea seedlings (Dong et al., 2017). For oil tea, drought stress decreased the photosynthetic rate, number of flower buds, fruit setting rate, fruit weight, and oil content of seeds, which seriously reduced the yield and economic benefits of oil tea orchards (Ding et al., 2017; Zhong et al., 2015). Therefore, to identify and develop more drought-tolerant cultivars of oil tea, an understanding of the physiological and metabolic responses to drought stress and the mechanism of tolerance is essential.

Metabolites are the final products of the cell regulation process, which is not only closely related to the growth and development of a plant, but also related to the impact of various environmental factors (Fiehn, 2002; Zhang et al., 2016). By identifying metabolites and metabolic pathways, the relationship between plants and the environment can be deeply understood from the metabolic level. It is common for 
researchers to compare the differences in metabolites of drought-resistant plants and drought-sensitive plants to determine the related mechanism of drought stress and select drought-tolerant cultivars (Guo et al., 2018; Miller et al., 2010). When facing long-term drought stress, maintaining intracellular osmotic balance is an important measure for plants to deal with drought stress (Drapal et al., 2017). The osmotic regulators are mainly small molecular organic compounds, including amino acids, organic acids, and soluble sugars (Evers et al., 2010). Some important small molecular metabolites, such as proline, salicylic acid, and sucrose, are known to accumulate in large quantities under drought stress, which can reduce the cytoplasmic water potential and maintain environmental stability (Amin et al., 2009; Chemikosova et al., 2006). Plant response to drought stress also involves a number of metabolic pathways, including glycolysis, tricarboxylic acid (TCA) cycle, sugar synthesis, and amino acid synthesis (Guo et al., 2018; Hare et al., 1998). The metabolic mechanisms of drought tolerance have been studied in many plant species; however, little is known about oil tea.

The traditional metabolite research has mainly focused on single metabolites (Marcek et al., 2019). With the development of analytical technology, gas chromatography-mass spectrometry (GC-MS) and liquid chromatography-mass spectrometry (LC-MS) have been widely used for tracking metabolomics changes (Zhang et al., 2016). GC-MS and LC-MS can determine both targeted and nontargeted metabolites, thus making it simple to identify more metabolites and to gain a better understanding of the regulation mechanism of plants under stress (Marcek et al., 2019; Zhang et al., 2016). In this study, 'Huajin' and 'Changlin53', which are two common cultivars, were used as experimental materials. Physiological and metabolic analyses and LC-MS were used to study the regulation mechanisms of oil tea under control conditions, moderate drought stress (MDS), and SDS.

\section{Materials and Methods}

Plant materials and drought stress treatments. The 2year-old seedlings of 'Huajin' and 'Changlin53' used in this experiment were obtained from a seedling breeding company (Shunfa Oil Tea Professional Cooperative, Zhuzhou, China). Healthy and uniform seedlings of the two cultivars were selected and planted in plastic pots $(20 \mathrm{~cm}$ diameter $\times 30 \mathrm{~cm}$ height) containing $8.0 \mathrm{~kg}$ of substrate, which was a mixture of equal volumes of acid red soil (classified as Lixisols in World Reference Base for Soil Resources), peat soil (Hongyue, Jiaxing, China), and perlite (Hongyue, Jiaxing, China). The seedlings were randomly placed in a greenhouse for acclimation for nearly 4 months (from 21 Feb. to 11 June 2017) before drought stress was initiated. During acclimation, the average air temperature in the greenhouse was $28.3^{\circ} \mathrm{C}$ during the day and $22.7^{\circ} \mathrm{C}$ at night. The average light intensity was $847 \mu \mathrm{mol} \cdot \mathrm{m}^{-2} \cdot \mathrm{s}^{-1}$. The average relative humidity was $47 \%$.

In this experiment, evapotranspiration (ET) of each plant, which was the total water loss through evaporation of water from soil surfaces and transpiration through stomata and epidermal pore, was determined to guide the irrigation to induce drought stress. Before initiation of the experiment, the acclimated seedlings ( $\approx 56-60 \mathrm{~cm}$ height and 84-90 leaves) of each cultivar were randomly divided into three groups, with each subjected to the control conditions, MDS, and SDS. Each treatment had 10 pot seedlings per cultivar. The drought stress experiment was initiated on 12 June 2017. After the initiation, three pots per treatment were randomly selected and weighted at 1800 HR every day; then, ET was calculated according to the difference between the two weights. Plants were irrigated every $3 \mathrm{~d}$ based on the cumulative ET. The control group was supplemented with $100 \%$ ET, the MDS group was supplemented with $70 \%$ ET, and the SDS group was supplemented with $30 \%$ ET. Drought stress treatment was continued for $60 \mathrm{~d}$ before termination.

Measurement of Photosynthesis. The photosynthetic parameters of oil tea leaves were measured on a sunny day from 0900 to $1100 \mathrm{HR}$ using a portable photosynthesis analyzer (LI6400; LI-COR Biosciences, Lincoln, NE) $53 \mathrm{~d}$ after the initiation of drought stress. Three seedlings of each treatment group were randomly selected for photosynthetic determination. The photosynthetic photon flux, $\mathrm{CO}_{2}$ concentration, and temperature of the leaf cuvette were set at $1000 \mu \mathrm{mol} \cdot \mathrm{m}^{-2} \cdot \mathrm{s}^{-1}$, $400 \mu \mathrm{mol} \cdot \mathrm{mol}^{-1}$, and $25{ }^{\circ} \mathrm{C}$, respectively. The net photosynthetic rate $\left(\mathrm{P}_{\mathrm{n}}\right), g_{\mathrm{S}}$, and transpiration rate $\left(\mathrm{T}_{\mathrm{r}}\right)$ were recorded when the environmental conditions in the cuvette were stable. The instantaneous water use efficiency (WUE) was calculated as $\mathrm{WUE}=\mathrm{P}_{\mathrm{n}} / \mathrm{T}_{\mathrm{r}}$ (Nijs et al., 1997).

Biochemical anAlyses. The leaves of oil tea were collected $60 \mathrm{~d}$ after the initiation of drought stress. The second leaves of the current year's branches of three individual seedlings per treatment were separately harvested and mixed well. One leaf of each mixture was selected to determine the chlorophyll content, and the others were stored at $-80{ }^{\circ} \mathrm{C}$ in a refrigerator to determine the malondialdehyde (MDA) and proline contents.

Fresh leaves with $1 \mathrm{~cm}^{2}$ were extracted in an ethanol-acetone mixture $(1: 1 \mathrm{v} / \mathrm{v})$ and the samples were placed in the dark at $4{ }^{\circ} \mathrm{C}$ for $24 \mathrm{~h}$; then, absorbance at 663 and $645 \mathrm{~nm}$ were read on a spectrophotometer. The concentrations of chlorophyll a (Chl a) and chlorophyll $\mathrm{b}(\mathrm{Chl} \mathrm{b})$ were calculated according to the formula of Arnon (1949). Proline content was determined using the acid ninhydrin colorimetric method according to Bates et al. (1973). The MDA content was assayed using the 2-thiobarbituric acid colorimetric method according to Jia et al. (2015).

Metabolite eXtraction AND PROFiling analyses. After 60 $\mathrm{d}$ of drought stress, the second leaves of the current year's branches of six individual seedlings per treatment were separately harvested and immediately transferred to liquid nitrogen for metabolic profiling.

The samples were homogenized with liquid nitrogen, and 20 $\mu \mathrm{L}$ of each sample was extracted with $120 \mu \mathrm{L}$ of precooled $50 \%$ methanol, vortexed for $1 \mathrm{~min}$, and incubated at room temperature for $10 \mathrm{~min}$. Then, the extraction mixture was stored overnight at $-20{ }^{\circ} \mathrm{C}$. After centrifugation at $4000 \mathrm{~g}_{\mathrm{n}}$ for $20 \mathrm{~min}$, the supernatants were used for LC-MS analysis. Additionally, quality control (QC) samples were prepared by combining 10 $\mu \mathrm{L}$ of each extract.

All chromatographic separations were performed using an ultra-performance liquid chromatography system (SCIEX, Foster City, CA). An ACQUITY BEH Amide column $(100 \mathrm{~mm} \times 2.1 \mathrm{~mm}$; particle size, $1.7 \mu \mathrm{m}$; Waters, Milford, MA) was used for the reverse-phase separation. The column oven was maintained at $35^{\circ} \mathrm{C}$. The flow rate was $0.4 \mathrm{~mL} \cdot \mathrm{min}^{-1}$ and the mobile phase consisted of solvent $\mathrm{A}$ (25 mM ammonium acetate $+25 \mathrm{mM} \mathrm{NH}_{4} \mathrm{H}_{2} \mathrm{O}$ ) and solvent $\mathrm{B}$ [isopropyl alcohol and cerium ammonium nitrate $(9: 1 \mathrm{v} / \mathrm{v})$ mixture containing $0.1 \%$ formic acid]. Gradient elution conditions were set as follows: 
0-0.5 min, $95 \% \mathrm{~B} ; 0.5-9.5 \mathrm{~min}, 95 \%$ to $65 \% \mathrm{~B} ; 9.5-10.5 \mathrm{~min}$, $65 \%$ to $40 \% \mathrm{~B} ; 10.5-12 \mathrm{~min}, 40 \% \mathrm{~B} ; 12-12.2 \mathrm{~min}, 40 \%$ to $95 \%$ $\mathrm{B}$; and $12.2-15 \mathrm{~min}, 95 \% \mathrm{~B}$. The injection volume for each sample was $4 \mu \mathrm{L}$. A high-resolution tandem mass spectrometer (Triple TOF 5600plus; SCIEX) was used to detect metabolites eluted from the column. During acquisition, the mass accuracy was calibrated for every 20 samples, and a QC sample was acquired after every 10 samples to evaluate the stability of LCMS.

EXPERIMENTAL DESIGN AND DATA ANALYSIS. The experiment had a randomized design with two factors. The data of photosynthesis and physiology indices were calculated using Excel (version 2013; Microsoft Corp., Redmond, WA). SPSS statistical software (version 22.0; IBM Corp., Armonk, NY) was used for statistical data analysis. All response variables were analyzed by two-way analysis of variance (ANOVA). The treatment differences were analyzed with the Tukey test $(P<$ $0.05)$. The difference between cultivars was compared with an independent samples $t$ test. Each treatment had three replicates.

The original data detected by LC-MS were preprocessed by XCMS software (version 3.4.1; SCIEX). The online Kyoto Encyclopedia of Genes and Genomes (KEGG) database was used to annotate the metabolites by matching the exact molecular mass data $(\mathrm{m} / \mathrm{z})$ of samples with those from the database (Kanehisa et al., 2014). Features that were detected in $<50 \%$ of QC samples or in $80 \%$ of biological samples were removed. The remaining peaks with missing values were imputed with the k nearest-neighbor algorithm. Then, all data of each sample were homogenized. The major metabolites were selected using Student's $t$ test $(P<0.05)$ if they had a variable importance in project (VIP) value $>1.0$ and ratio $>2.0$ or $<0.5$. SIMCA-P software (version 14.1; Umetrics, Umea, Sweden) was used to create a principal component analysis (PCA) and loading plot. Subsequently, the metabolic pathway was constructed using Visio (version 2013; Microsoft Corp.) and the KEGG metabolic database as a reference.

\section{Results}

Physiological Responses to Drought stress. Both cultivars showed significant decreases in $\mathrm{P}_{\mathrm{n}}, g_{\mathrm{S}}$, and $\mathrm{T}_{\mathrm{r}}$, especially under SDS, compared with those under control conditions (Fig. $1 \mathrm{~A}-\mathrm{C}) . \mathrm{P}_{\mathrm{n}}$ of 'Changlin53' was greater than that of 'Huajin' under SDS, but there was no difference in the $g_{S}$ and $T_{r}$ of the cultivars. WUE was increased in both cultivars under drought stress (Fig. 1D). Leaves of 'Changlin53' had greater WUE than 'Huajin' under both drought stress conditions. The Chl a content of 'Huajin' leaves decreased significantly with both drought stress treatments, but there was no statistical difference exhibited by 'Changlin53' (Fig. 1E). The Chl b content decreased under SDS in 'Huajin' leaves, but there was no significant decrease in 'Changlin53' leaves (Fig. 1F). The Chl a and Chl b contents of 'Changlin53' leaves were greater than those of 'Huajin' leaves under both drought stress conditions. The proline contents of 'Changlin53' and 'Huajin' increased with both drought stress treatments (Fig. 1G). The proline content of 'Huaijn' under SDS decreased compared with that under MDS. Leaves of 'Changlin53' had a greater proline content under SDS compared with the leaves of 'Huajin'. The MDA content of 'Huajin' leaves increased with both levels of drought stress (Fig. 1H), and it was greater than that of 'Changlin53' leaves with both levels of drought stress. These results indicated that 'Huajin' is less tolerant to drought stress, especially SDS, compared with 'Changlin53'.

Metabolic Profiles. A total of 41 metabolites, including 12 amino acids, 12 organic acids, 10 sugar alcohols, 3 fatty acids, and 4 phenols, were unequivocally identified and their relative concentrations were determined (Table 1). PCA is an unsupervised pattern recognition technique that can easily provide an overview of the differences of multivariate datasets using dimension reduction analysis. The PCA results showed that the six biological replicates of each treatment were gathered together, whereas the cultivars and treatments were obviously separated (Fig. 2A). The first principal component (PC1) and second principal component (PC2) represent 52.9\% and 20.8\%, respectively, of the variations among the samples. The contribution of metabolites for PC1 were dominated by salicylic acid, hydroxycinnamic acid, galactose, shikimic acid, coumarin, and chlorogenic acid, whereas succinic acid, proline, pyroglutamic acid, ribose, asparagine, aspartic acid, citraconic acid, quinic acid, melibiose, maltose, and sucrose were the major contributors of PC2 (Fig. 2B).

Metabolic Changes in ReSPonse to Drought Stress. After 60 d under MDS, 32 and 16 metabolites in leaves of 'Huajin' and 'Changlin53' were significantly changed (Table 2). Most carbohydrates (glucose, maltose, mannose, melibiose, sucrose, and inositol) were accumulated in 'Changlin53' leaves under MDS. Maltose, melibiose, and sucrose were decreased in 'Huajin' leaves, whereas others, including allose, erythrose-4phosphate, glucose, inositol, mannose, and ribose, were increased (Table 2). Some amino acids on the glutamic acidmediated proline biosynthesis pathway, including glutamic acid, arginine, and proline, were increased in both cultivars, whereas glutamine and pyroglutamic acid were increased in 'Huajin' leaves (Table 2, Fig. 3). Aspartic acid, asparagine, threonine, and norvaline were increased in 'Huajin' leaves, whereas phenylalanine and tryptophan were increased in 'Changlin53' leaves. The MDS caused reductions in phenylalanine, quinic acid, and shikimic acid in 'Huajin' leaves. Chlorogenic acid, coumarin, phloretin, and syringing, the secondary metabolites of the shikimic acid metabolic pathway in 'Huajin' leaves, were simultaneously decreased (Table 2, Fig. 3). Pyruvic acid, which is the intermediate product of glycolysis, was increased in 'Huajin' leaves in MDS. Succinic acid and fumaric acid, which are TCA cycle intermediates, were increased in 'Huajin' leaves under MDS. Citraconic acid, maleic acid, and pantothenate were increased in 'Huajin' leaves, and maleic acid and salicylic acid were increased in 'Changlin53' leaves. Unsaturated fatty acids including cis-9palmitoleic acid and oleic acid were increased in 'Huajin' leaves, and arachidonic acid was increased in 'Changlin53' leaves.

There were 21 and 22 significantly changed metabolites in 'Huajin' and 'Changlin53' leaves under SDS, respectively (Table 2). Most sugar alcohols were decreased in 'Huajin' and 'Changlin53' leaves under SDS (Table 2). Accumulation of some amino acids, including arginine, aspartic acid, asparagine, citrulline, norvaline, pyroglutamic acid, proline, and tryptophan, were significantly increased in 'Changlin53' leaves, whereas four amino acids, including arginine, glutamine, norvaline, and threonine, were increased in 'Huajin' leaves, and five amino acids, including asparagine, aspartic acid, citrulline, phenylalanine, and pyroglutamic acid, were decreased in 'Huajin' leaves. The SDS also caused reductions in 

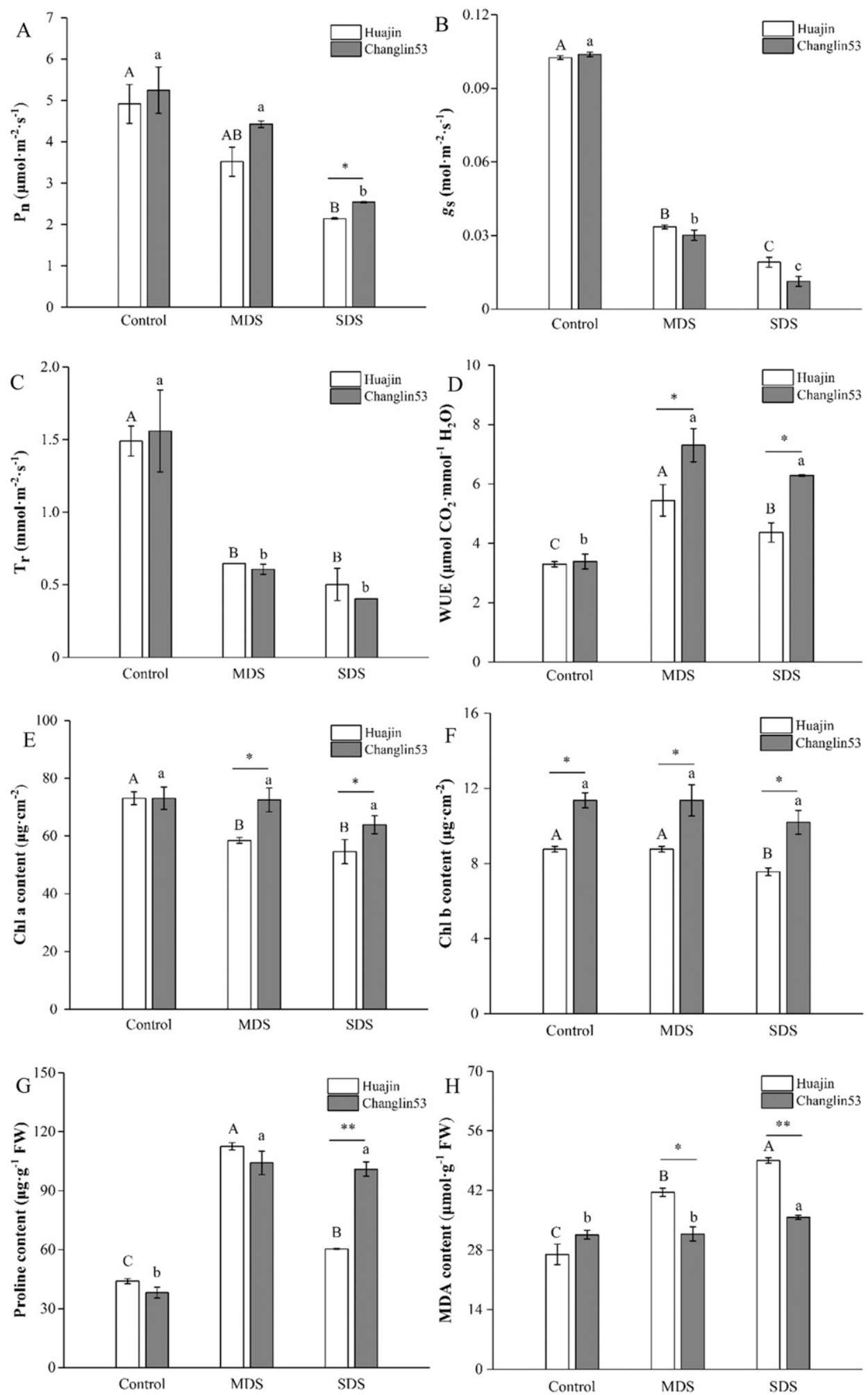

Fig. 1. Effects of drought stress on photosynthetic and physiological activities in leaves of two oil tea cultivars (Huajin and Changlin53). The two cultivars were treated with control conditions, moderate drought stress (MDS), and severe drought stress (SDS). (A) The net photosynthetic rate $\left(\mathrm{P}_{\mathrm{n}}\right),(\mathbf{B})$ stomatal conductance $\left(g_{\mathrm{S}}\right)$, and $(\mathbf{C})$ transpiration rate $\left(\mathrm{T}_{\mathrm{r}}\right)$ were measured on a sunny day, $53 \mathrm{~d}$ after the initiation of drought stress. (D) Water use efficiency (WUE) was calculated as $\mathrm{P}_{\mathrm{n}} / \mathrm{T}_{\mathrm{r}}$. (E) Chlorophyll a (Chl a), (F) chlorophyll b (Chl b), (G) proline, and (H) malondialdehyde (MDA) were determined after $60 \mathrm{~d}$ of drought stress. The experiment included three biological replicates. Different letters on the same cultivar indicate significant differences $(P<0.05)$ among treatments; * and ** represent significant differences at $P<0.05$ and $P<0.01$ between cultivars in the same treatment. organic acids, especially shikimic acid, hydroxycinnamic acid, and chlorogenic acid in 'Huajin' leaves, whereas salicylic acid was significantly increased and quinic acid, hydroxycinnamic acid were decreased in 'Changlin53' leaves (Table 2, Fig. 3). Citraconic acid, maleic acid, and mevalonic acid also increased in 'Changlin53' leaves. Cis-9-palmitoleic acid (unsaturated fatty acid) was increased in 'Huajin' and 'Changlin53' leaves. Phenols, including coumarin, luteolin, phloretin, and syringing, were reduced; this reduction was greater in 'Huajin' than in 'Changlin53'.

\section{Discussion}

The rate of photosynthesis of leaves usually decreased with increasing drought stress (Guo et al., 2018; Marcińska et al., 2013). Our results showed that $\mathrm{P}_{\mathrm{n}}, g_{\mathrm{S}}$, and $\mathrm{T}_{\mathrm{r}}$ decreased significantly under drought stress. Similar results were reported for apple (Malus pumila) trees; the transpiration rate and photosynthetic rate were reduced by the decreased $g_{\mathrm{S}}$, thereby protecting the tissue water status under drought stress (Wang et al., 2018). WUE increased significantly under drought stress, and the WUE of 'Changlin53' was greater than that of 'Huajin'. This was consistent with the results of another study (Marcińska et al., 2013) in which drought-resistant plants led to greater WUE. The contents of $\mathrm{Chl}$ a and $\mathrm{Chl} \mathrm{b}$ in 'Huajin' leaves decreased under drought stress, and the contents of Chl a and Chl b in 'Huajin' leaves were lower than those in 'Changlin53' leaves under both drought stress types. The chlorophyll content of drought-susceptible plants decreased significantly under drought stress, which may have occurred through the degradation of chlorophyll to reduce the light harvesting ability and to reduce the excessive production of reactive oxygen species caused by excessive energy (Mafakheri et al., 2010; Marcińska et al., 2013). Maintaining a high proline content can maintain cell turgidity, scavenge reactive oxygen species, and maintain the stability of phospholipid, nucleic 
Table 1. Relative concentration of 41 key metabolites in the leaves of two oil tea cultivars, Huajin and Changlin53, after drought stress treatments for $60 \mathrm{~d}$.

\begin{tabular}{|c|c|c|c|c|c|c|c|}
\hline \multirow[b]{3}{*}{ No. } & \multirow[b]{3}{*}{ Metabolites } & \multicolumn{6}{|c|}{ Relative concn (mean \pm SD) } \\
\hline & & \multicolumn{3}{|c|}{ Huajin } & \multicolumn{3}{|c|}{ Changlin53 } \\
\hline & & SDS & MDS & Control & SDS & MDS & Control \\
\hline & Amino acids & & & & & & \\
\hline 2 & Asparagine & $2.55 \pm 0.27$ & $12.29 \pm 1.24$ & $5.29 \pm 0.65$ & $10.00 \pm 0.74$ & $5.94 \pm 0.23$ & $4.04 \pm 0.35$ \\
\hline 3 & Aspartic acid & $1.01 \pm 0.35$ & $7.31 \pm 0.92$ & $2.47 \pm 0.22$ & $5.07 \pm 0.31$ & $3.27 \pm 0.16$ & $1.62 \pm 0.12$ \\
\hline 4 & Citrulline & $0.25 \pm 0.03$ & $0.70 \pm 0.15$ & $0.59 \pm 0.04$ & $0.60 \pm 0.04$ & $0.20 \pm 0.02$ & $0.22 \pm 0.01$ \\
\hline 6 & Glutamine & $2.55 \pm 0.62$ & $1.77 \pm 0.06$ & $0.69 \pm 0.06$ & $0.55 \pm 0.05$ & $0.62 \pm 0.05$ & $0.65 \pm 0.19$ \\
\hline 7 & Norvaline & $1.25 \pm 0.09$ & $1.94 \pm 0.07$ & $0.61 \pm 0.03$ & $0.89 \pm 0.03$ & $0.46 \pm 0.04$ & $0.43 \pm 0.04$ \\
\hline 8 & Phenylalanine & $0.09 \pm 0.01$ & $0.10 \pm 0.01$ & $0.20 \pm 0.05$ & $0.58 \pm 0.12$ & $1.71 \pm 0.27$ & $0.45 \pm 0.06$ \\
\hline 9 & Proline & $5.97 \pm 1.61$ & $19.99 \pm 3.47$ & $10.19 \pm 6.26$ & $10.40 \pm 0.60$ & $12.53 \pm 0.85$ & $5.12 \pm 0.33$ \\
\hline 10 & Pyroglutamic acid & $1.25 \pm 0.18$ & $13.75 \pm 1.51$ & $4.96 \pm 0.35$ & $6.84 \pm 0.53$ & $4.00 \pm 0.50$ & $3.39 \pm 0.37$ \\
\hline 11 & Threonine & $10.77 \pm 2.10$ & $11.59 \pm 1.08$ & $4.87 \pm 0.35$ & $8.07 \pm 0.65$ & $11.88 \pm 0.87$ & $11.77 \pm 0.86$ \\
\hline 3 & Fumaric acid & $1.65 \pm 0.12$ & $2.85 \pm 0.39$ & $1.41 \pm 0.18$ & $1.14 \pm 0.10$ & $1.02 \pm 0.10$ & $1.77 \pm 0.24$ \\
\hline 4 & Hydroxycinnamic acid & $28.37 \pm 4.50$ & $69.52 \pm 4.40$ & $85.68 \pm 6.67$ & $7.94 \pm 0.56$ & $15.33 \pm 1.44$ & $19.97 \pm 2.26$ \\
\hline 5 & Maleic acid & $4.11 \pm 0.46$ & $6.55 \pm 0.57$ & $2.95 \pm 0.48$ & $1.80 \pm 0.22$ & $3.68 \pm 0.20$ & $0.87 \pm 0.10$ \\
\hline 6 & Mevalonic acid & $0.90 \pm 0.14$ & $0.91 \pm 0.09$ & $1.30 \pm 0.10$ & $2.89 \pm 0.84$ & $1.28 \pm 0.10$ & $0.96 \pm 0.11$ \\
\hline 7 & Pantothenate & $0.30 \pm 0.02$ & $1.22 \pm 0.10$ & $0.39 \pm 0.02$ & $0.30 \pm 0.03$ & $0.35 \pm 0.01$ & $0.27 \pm 0.02$ \\
\hline 8 & Pyruvic acid & $0.10 \pm 0.02$ & $0.58 \pm 0.10$ & $0.11 \pm 0.01$ & $0.40 \pm 0.03$ & $0.40 \pm 0.09$ & $0.24 \pm 0.03$ \\
\hline 9 & Quinic acid & $15.34 \pm 2.21$ & $8.73 \pm 0.29$ & $17.94 \pm 2.09$ & $4.58 \pm 0.55$ & $7.04 \pm 0.65$ & $9.96 \pm 0.56$ \\
\hline 10 & Salicylic acid & $2.55 \pm 0.37$ & $2.28 \pm 0.32$ & $2.31 \pm 0.21$ & $116.52 \pm 22.95$ & $53.98 \pm 7.37$ & $26.26 \pm 2.95$ \\
\hline 11 & Shikimic acid & $12.96 \pm 2.50$ & $8.36 \pm 0.63$ & $23.07 \pm 2.27$ & $4.38 \pm 0.38$ & $4.24 \pm 0.40$ & $3.64 \pm 0.33$ \\
\hline \multirow[t]{2}{*}{12} & Succinic acid & $11.58 \pm 1.72$ & $40.26 \pm 4.63$ & $9.22 \pm 1.36$ & $8.92 \pm 3.26$ & $7.59 \pm 1.10$ & $14.15 \pm 6.64$ \\
\hline & Carbohydrates & & & & & & \\
\hline 8 & Melibiose & $7.51 \pm 0.21$ & $8.28 \pm 0.48$ & $23.05 \pm 1.11$ & $0.65 \pm 0.05$ & $14.72 \pm 0.57$ & $3.85 \pm 0.25$ \\
\hline 9 & Ribose & $8.92 \pm 0.63$ & $16.79 \pm 0.61$ & $7.63 \pm 0.58$ & $10.45 \pm 0.92$ & $11.00 \pm 0.21$ & $9.92 \pm 0.43$ \\
\hline \multirow[t]{2}{*}{10} & Sucrose & $1.69 \pm 0.05$ & $1.84 \pm 0.18$ & $5.80 \pm 0.24$ & $1.75 \pm 0.02$ & $18.76 \pm 0.92$ & $4.28 \pm 0.09$ \\
\hline & Fatty acids & & & & & & \\
\hline 1 & Arachidonic acid & $1.35 \pm 0.18$ & $1.05 \pm 0.06$ & $1.42 \pm 0.14$ & $1.41 \pm 0.26$ & $2.18 \pm 0.31$ & $1.00 \pm 0.04$ \\
\hline 2 & Cis-9-palmitoleic acid & $1.56 \pm 0.13$ & $1.54 \pm 0.09$ & $0.70 \pm 0.06$ & $2.47 \pm 0.15$ & $1.40 \pm 0.10$ & $0.83 \pm 0.06$ \\
\hline \multirow[t]{2}{*}{3} & Oleic acid & $3.46 \pm 0.59$ & $5.80 \pm 1.77$ & $1.91 \pm 0.25$ & $1.04 \pm 0.11$ & $1.50 \pm 0.08$ & $1.79 \pm 0.14$ \\
\hline & Phenols & & & & & & \\
\hline 1 & Coumarin & $16.93 \pm 3.91$ & $17.42 \pm 7.67$ & $45.14 \pm 2.44$ & $3.31 \pm 0.24$ & $6.05 \pm 0.41$ & $7.17 \pm 0.52$ \\
\hline 2 & Luteolin & $1.02 \pm 0.07$ & $2.66 \pm 0.12$ & $2.66 \pm 0.15$ & $1.42 \pm 0.27$ & $1.42 \pm 0.13$ & $1.13 \pm 0.38$ \\
\hline 3 & Phloretin & $1.64 \pm 0.41$ & $1.68 \pm 0.15$ & $4.42 \pm 0.74$ & $5.97 \pm 1.35$ & $5.69 \pm 0.93$ & $6.70 \pm 1.23$ \\
\hline 4 & Syringin & $0.29 \pm 0.03$ & $0.33 \pm 0.02$ & $0.74 \pm 0.11$ & $0.14 \pm 0.03$ & $0.17 \pm 0.06$ & $0.40 \pm 0.06$ \\
\hline
\end{tabular}

Treatments include control conditions, moderate drought stress (MDS), and severe drought stress (SDS). The relative concentrations of metabolites in leaves of each treatment were determined by liquid chromatography-mass spectrometry. The metabolites listed in this table were significantly changed under MDS or SDS compared with control conditions. These major metabolites were selected using Student's $t$ test $(P<$ 0.05 ), VIP value $>1.0$, and ratio $>2.0$ or $<0.5$. Each treatment had six biological replicates.

acid, protein, and other macromolecular substances (Hare et al., 1998; Maggio et al., 2002; Marcińska et al., 2013). The content of proline in 'Changlin53' under SDS was significantly greater than that in 'Huajin', which meant that 'Changlin53' may have a more stable intracellular environment. The MDA content of
'Huajin' increased significantly with increased drought stress, and it was significantly greater than that of 'Changlin 53 '. MDA formation is the result of the peroxidation of the membrane lipid, and the increased MDA content indicates that the cell membrane structure is destroyed (Chaoui et al., 1997). 

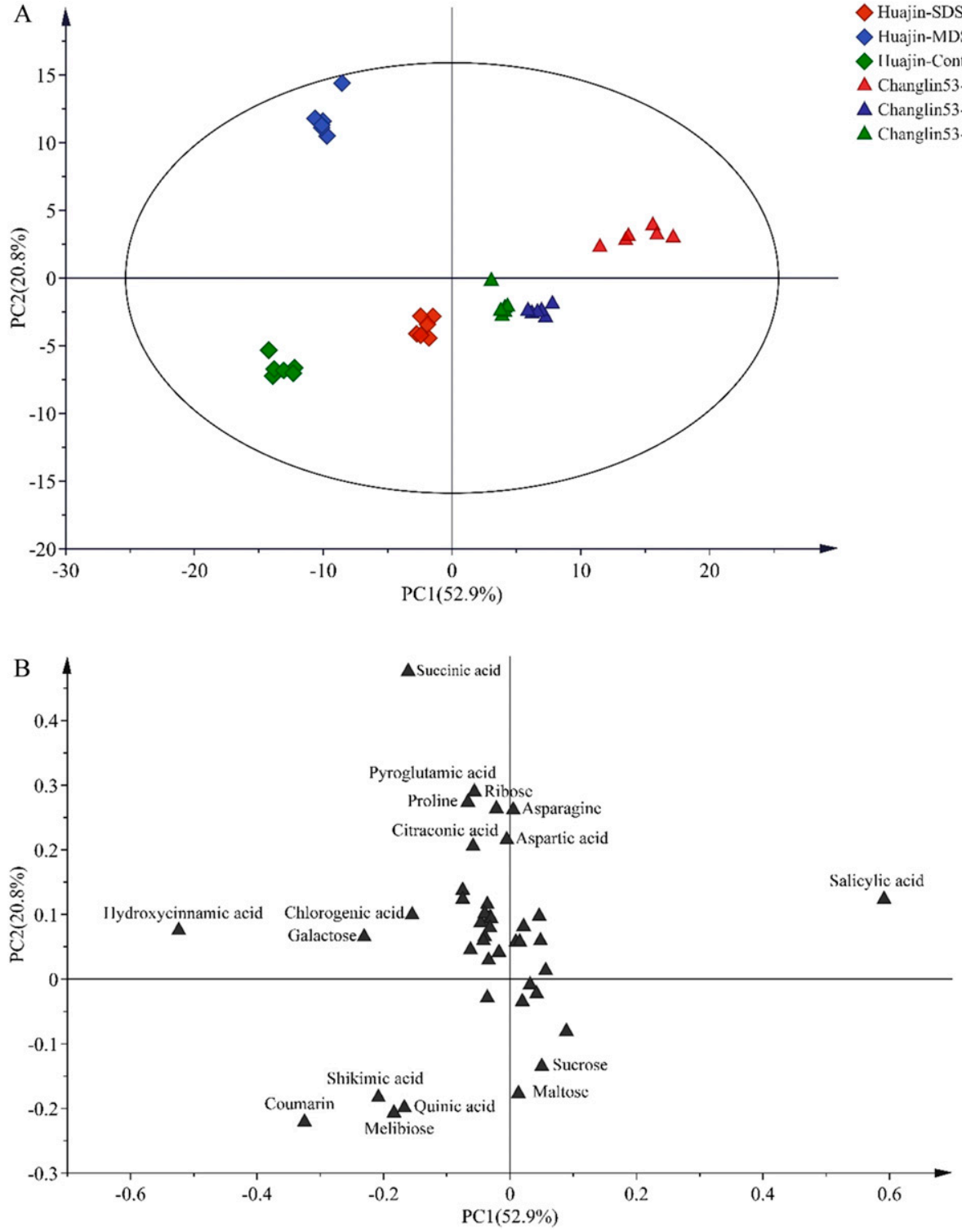

Fig. 2. (A) The principal component analysis (PCA) and its (B) loading plot of the metabolic profiles in leaves of two oil tea cultivars (Huajin and Changlin53) after control conditions, moderate drought stress (MDS), and severe drought stress (SDS) treatments for $60 \mathrm{~d}$. Each treatment had six biological replicates.
$\Delta$ Changlin53-Control

amino acids, organic acids, and other substances that are more effective for regulating osmotic balance (Todaka et al., 2017; Yang et al., 2017).

Amino acids are precursors of protein synthesis that have an important role in resisting drought stress (Fabregas and Fernie, 2019). Proline is a key osmotic regulator, and its accumulation under drought stress has been reported for many plants; this can reduce cell osmotic potential and water evaporation and maintain a stable intracellular environment (Mafakheri et al., 2010). Our results showed that MDS significantly increased various amino acids in two cultivars of oil tea, among which the glutamate-mediated proline biosynthesis pathway was enhanced, and glutamic acid, proline, arginine, and other amino acids in this pathway were significantly increased (Fig. 3). Under SDS, the content of proline in 'Huajin' was decreased, whereas the content of proline in 'Changlin53' was still significantly increased, which was consistent with the results of our biochemical test (Fig. 1G). The upregulation of the glutamic acid synthesis pathway eventually increased the accumulation of proline (Zhang et al., 2011). Under SDS, pyroglutamic acid, citrulline, and arginine in the glutamate-mediated biosynthesis pathway of 'Changlin53' continued to increase, which provided sufficient upstream metabolites for proline biosynthesis. Although glutamine and arginine were still increased in 'Huajin' under SDS, the contents of pyroglutamic acid

Many studies have shown that carbohydrates accumulate rapidly early during drought stress, which not only ensures an adequate carbohydrate supply but also can be used as the main osmotic regulator to resist drought stress (Fabregas and Fernie, 2019; Todaka et al., 2017). Our results showed that the carbohydrates in two cultivars of oil tea were increased under MDS, including glucose, mannose, ribose, allose, and inositol, whereas the contents of sucrose, melibiose, and maltose in 'Huajin' leaves were decreased. It is possible that those disaccharides are hydrolyzed to glucose, which is used more in glycolysis and the TCA cycle to enhance plant metabolism and resist drought stress (Bogdan and Zagdanska, 2006). However, under SDS, various carbohydrates of oil tea were significantly decreased. It has been suggested that carbohydrates have an osmotic regulatory role in plants under MDS; however, under SDS, carbohydrates are not the main osmotic regulators, and more carbohydrates are used to synthesize and citrulline were significantly decreased, indicating that the glutamic acid biosynthesis pathway of 'Huajin' weakened and finally decreased the proline content under SDS. In addition, the contents of tryptophan, aspartic acid, and asparagine in 'Changlin53' were significantly increased, whereas those in 'Huajin' were decreased, indicating that 'Changlin53' has more potential to decrease the osmotic potential of cells by increasing the amino acid content under SDS to resist drought stress.

The responses of organic acids metabolite of two cultivars of oil tea were different under drought stress. Quinic acidmediated and shikimic acid-mediated secondary metabolites synthesis were decreased under both types of drought stress in 'Huajin' leaves. Those results showed that the synthesis ability of secondary metabolites was reduced in 'Huajin' leaves. Under SDS, quinic acid and hydroxycinnamic acid decreased significantly in 'Changlin53', but the salicylic acid content continued to increase significantly under MDS and SDS. It is possible that 
Table 2. Fold changes of 41 key metabolites in leaves of two oil tea cultivars, Huajin and Changlin53, after drought stress treatments for $60 \mathrm{~d}$.

\begin{tabular}{|c|c|c|c|c|}
\hline \multirow[b]{3}{*}{ Metabolite name } & \multicolumn{4}{|c|}{ Fold changes } \\
\hline & \multicolumn{2}{|c|}{ Huajin } & \multicolumn{2}{|c|}{ Changlin53 } \\
\hline & $\log _{2}($ SDS/control $)$ & $\log _{2}(\mathrm{MDS} /$ control $)$ & $\log _{2}($ SDS/control $)$ & $\log _{2}{ }^{(\mathrm{MDS} / \text { control })}$ \\
\hline \multicolumn{5}{|l|}{ Amino acids } \\
\hline Arginine & $1.43^{z}$ & 3.93 & 1.36 & 1.39 \\
\hline Asparagine & $-\underline{1.05}$ & 1.21 & 1.31 & 0.56 \\
\hline Aspartic acid & -1.29 & 1.57 & 1.64 & 1.01 \\
\hline Citrulline & -1.27 & 0.23 & 1.46 & -0.10 \\
\hline Glutamic acid & -0.36 & 1.34 & 0.72 & 1.78 \\
\hline Glutamine & 1.79 & 1.36 & -0.23 & -0.07 \\
\hline Norvaline & 1.03 & 1.67 & 1.05 & 0.09 \\
\hline Phenylalanine & -1.17 & -1.06 & 0.36 & 1.92 \\
\hline Proline & $-\overline{-0.36}$ & $\overline{1.45}$ & 1.02 & 1.29 \\
\hline Pyroglutamic acid & $-\underline{1.99}$ & 1.47 & 1.01 & 0.24 \\
\hline Threonine & $\overline{1.15}$ & 1.25 & -0.55 & 0.01 \\
\hline Tryptophan & -0.19 & -0.12 & 1.29 & 2.11 \\
\hline \multicolumn{5}{|l|}{ Organic acids } \\
\hline Citraconic acid & 0.03 & 1.04 & 1.15 & 0.30 \\
\hline Chlorogenic acid & $-\underline{1.20}$ & 0.05 & 0.08 & -0.61 \\
\hline Fumaric acid & $\overline{0.22}$ & 1.02 & -0.63 & -0.80 \\
\hline Hydroxycinnamic acid & $-\underline{1.59}$ & -0.30 & $-\underline{1.33}$ & -0.38 \\
\hline Maleic acid & 0.48 & 1.15 & 1.05 & 2.08 \\
\hline Mevalonic acid & -0.54 & -0.53 & 1.59 & 0.42 \\
\hline Pantothenate & -0.41 & 1.38 & 0.14 & 0.34 \\
\hline Pyruvic acid & -0.08 & 2.40 & 0.76 & 0.75 \\
\hline Quinic acid & -0.23 & $-\underline{1.04}$ & $-\underline{1.12}$ & -0.50 \\
\hline Salicylic acid & 0.14 & $-\overline{-0.02}$ & $\overline{2.15}$ & 1.04 \\
\hline Shikimic acid & -1.07 & -1.44 & 0.27 & 0.22 \\
\hline Succinic acid & $\overline{0.36}$ & $\overline{2.15}$ & -0.67 & -0.90 \\
\hline \multicolumn{5}{|l|}{ Carbohydrates } \\
\hline Allose & 0.18 & 1.26 & -0.08 & 0.12 \\
\hline Erythrose 4-phosphate & $-\underline{-1.43}$ & 1.17 & -0.11 & -0.42 \\
\hline Inositol & $-\overline{0.46}$ & 1.25 & 1.11 & 1.00 \\
\hline Galactose & -0.81 & 0.06 & $-\underline{-1.23}$ & 0.04 \\
\hline Glucose & -0.10 & 1.07 & -0.06 & 1.06 \\
\hline Maltose & $-\underline{1.34}$ & $-\underline{1.40}$ & $-\underline{1.32}$ & 1.35 \\
\hline Mannose & 0.90 & 1.28 & -0.58 & 1.19 \\
\hline Melibiose & $-\underline{1.62}$ & $-\underline{1.48}$ & $-\underline{2.56}$ & 1.94 \\
\hline Ribose & $\overline{0.23}$ & $\overline{1.14}$ & $\overline{0.08}$ & 0.15 \\
\hline Sucrose & $-\underline{1.78}$ & $-\underline{1.66}$ & $-\underline{1.29}$ & 2.13 \\
\hline \multicolumn{5}{|l|}{ Fatty acids } \\
\hline Arachidonic acid & -0.07 & -0.43 & 0.49 & 1.12 \\
\hline Cis-9-palmitoleic acid & 1.15 & 1.13 & 1.57 & 0.75 \\
\hline Oleic acid & 0.86 & 1.60 & -0.78 & -0.25 \\
\hline \multicolumn{5}{|l|}{ Phenols } \\
\hline Coumarin & -1.41 & -1.37 & $-\underline{1.11}$ & -0.25 \\
\hline Luteolin & $-\overline{1.38}$ & $-\overline{-0.02}$ & $\overline{0.33}$ & 0.33 \\
\hline Phloretin & $-\overline{1.43}$ & -1.39 & -0.17 & -0.24 \\
\hline Syringin & $-\overline{1.38}$ & $-\overline{1.18}$ & $-\underline{1.50}$ & $-\underline{1.26}$ \\
\hline
\end{tabular}

${ }^{\mathrm{z} B o l d}$ numbers represent significantly increased values, and italic underlined numbers represent significantly decreased values.

The treatments were control conditions, moderate drought stress (MDS), and severe drought stress (SDS). The relative concentrations of metabolites in leaves of each treatment group were determined by liquid chromatography-mass spectrometry. The significantly changed metabolites were selected using Student's $t$ test $(P<0.05)$, VIP value $>1.0$, and ratio $>2.0$ or $<0.5$. The fold changes were calculated according to the relative concentration using the formula $\log _{2}$ (drought/control).
'Changlin53' can reduce the synthesis of secondary metabolites of the shikimic acid pathway, and that more upstream metabolites were used in the synthesis of salicylic acid. Salicylic acid, as an endogenous signal molecule in plants, can protect pigments from injury, improve the antioxidant system, remove excess reactive oxygen species, and reduce the level of membrane peroxide, thereby alleviating the inhibition of stress on plant growth and improvement in the metabolic activity of cells (Aim and Mahmood, 2011; Amin et al., 2009; Kang et al., 2013). In this study, the content of salicylic acid in 'Changlin53' was greater than that in 'Huajin' (Table 1), which indicated that the accumulation of salicylic acid was an important mechanism of 'Changlin53' against drought stress. Under MDS, the contents of pyruvic acid, succinic acid, and fumaric acid in 'Huajin' were increased, which indicated that the glycolysis and TCA cycle were enhanced, the production of ATP was accelerated, and the physiological metabolism was promoted to resist stress environments (Yang et al., 2017).

Our results showed that the unsaturated fatty acids were significantly increased under drought stress. Fatty acid metabolism is an important part of biological metabolism and has an important role in plant resistance. Many studies have shown that stress can lead to the destruction of the cell membrane structure and spillage of cell contents (Salama and Mansour, 2015). Plants can maintain plasma membrane mobility by increasing the ratio of unsaturated fatty acids to saturated fatty acids (Mansour, 2013; Salama and Mansour, 2015). Therefore, increasing the contents of unsaturated fatty acids in oil tea leaves under drought stress is an effective way to cope with stress.

The responses of two cultivars of oil tea to drought stress were examined physiologically and metabolically, and the differences in tolerance to drought stress between the two cultivars of oil tea were determined. Under MDS, 'Changlin53' resisted drought stress by 


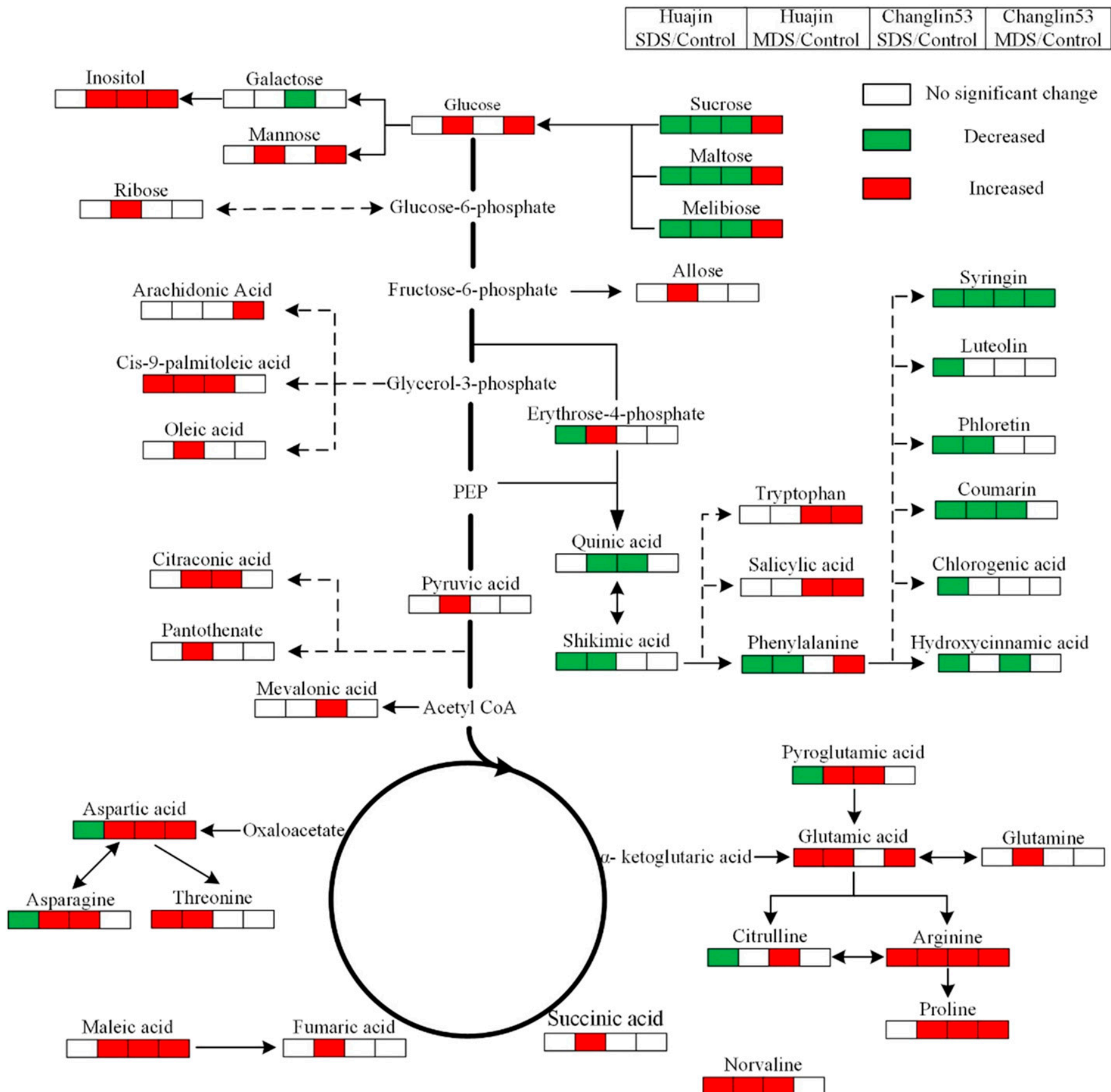

Fig. 3. Pathway display of significant changes in metabolite levels in leaves of oil tea seedlings of two cultivars (Huajin and Changlin53) after moderate drought stress (MDS) and severe drought stress (SDS) compared with control conditions after treatment for $60 \mathrm{~d}$. Red boxes represent significant increases in metabolites. Green boxes represent significant decreases in metabolites.

increasing the contents of carbohydrates, amino acids, and some organic acids; under SDS, the contents of soluble sugars were decreased, and more of them were used for the synthesis of amino acids and organic acids. The glutamic acid-mediated biosynthesis pathway and salicylic acid synthesis were continuously upregulated in 'Changlin53' under MDS and SDS, which could maintain the osmotic balance and stable intercellular environment. Under MDS, the contents of amino acids, monosaccharide, and organic acids increased in 'Huajin' leaves, and the shikimic acid-mediated secondary metabolite synthesis pathway was weakened; more amino acids, monosaccharide, and organic acids were used to increase glycolysis and the TCA cycle to accelerate energy production and enhance the glutamic acid-mediated biosynthesis pathway to increase the osmotic regulation ability. Under SDS, the contents of carbohydrates, organic acids, and some amino acids were decreased, indicating serious damage in 'Huajin' by SDS. This study provided an important theoretical basis for the selection of drought-resistant oil tea cultivars and will help improve the water management of oil tea seedlings. 


\section{Literature Cited}

Aim, B. and H. Mahmood. 2011. Effect of drought stress and its interaction with ascorbate and salicylic acid on okra (Hibiscus esculents L.) germination and seedling growth. J. Stress Physiol. Biochem. 7:55-65.

Amin, B., G. Mahleghah, H.M.R. Mahmood, and M. Hossein. 2009. Evaluation of interaction effect of drought stress with ascorbate and salicylic acid on some of physiological and biochemical parameters in okra (Hibiscus esculentus L.). Res. J. Biol. Sci. 4:380-387.

Arnon, D.I. 1949. Copper enzymes in isolated chloroplasts: Polyphenoloxidase in Beta vulgaris. Plant Physiol. 24:1-15.

Bates, L.S., R.P. Waldren, and I.D. Teare. 1973. Rapid determination of free proline for water-stress studies. Plant Soil 39:205-207.

Bogdan, J. and B. Zagdanska. 2006. Changes in the pool of soluble sugars induced by dehydration at the heterotrophic phase of growth of wheat seedlings. Plant Physiol. Biochem. 44:787-794.

Chaoui, A., S. Mazhoudi, M.H. Ghorbal, and E. Ferjani. 1997. Cadmium and zinc induction of lipid peroxidation and effects on antioxidant enzyme activities in bean (Phaseolus vulgaris L.). Plant Sci. 127:139-147.

Chemikosova, S.B., N.V. Pavlencheva, O.P. Gur'yanov, and T.A. Gorshkova. 2006. The effect of soil drought on the phloem fiber development in long-fiber flax. Russ. J. Plant Physiol. 53:656-662.

Ding, S., Q. Zhong, T. Yuan, L. Cao, C. Yan, Y. Yuan, X. Zhang, and J. Lin. 2017. Effects of drought stress on Camellia oleifera flower-bud growth and production. J. Nanjing For. Univ. 41:197-202.

Dong, B., B. Wu, W. Hong, X. Li, Z. Li, L. Xue, and Y. Huang. 2017. Transcriptome analysis of the tea oil camellia (Camellia oleifera) reveals candidate drought stress genes. PLoS One 12:e0181835.

Drapal, M., E.R. Farfan-Vignolo, O.R. Gutierrez, M. Bonierbale, E. Mihovilovich, and P.D. Fraser. 2017. Identification of metabolites associated with water stress responses in Solanum tuberosum L. clones. Phytochemistry 135:24-33.

Evers, D., I. Lefevre, S. Legay, D. Lamoureux, J.F. Hausman, R.O. Rosales, L.R. Marca, L. Hoffmann, M. Bonierbale, and R. Schafleitner. 2010. Identification of drought-responsive compounds in potato through a combined transcriptomic and targeted metabolite approach. J. Expt. Bot. 61:2327-2343.

Fabregas, N. and A.R. Fernie. 2019. The metabolic response to drought. J. Expt. Bot. 70:1077-1085.

Fiehn, O. 2002. Metabolomics the link between genotypes and phenotypes. Plant Mol. Biol. 48:155-171.

Guo, R., L. Shi, Y. Jiao, M. Li, X. Zhong, F. Gu, Q. Liu, X. Xia, and H. Li. 2018. Metabolic responses to drought stress in the tissues of drought-tolerant and drought-sensitive wheat genotype seedlings. AoB Plants 10:ply016.

Hare, P.D., W.A. Cress, and J. Van Staden. 1998. Dissecting the roles of osmolyte accumulation during stress. Plant Cell Environ. 21:535553.

Jaleel, C.A., P. Manivannan, A. Wahid, M. Farooq, R. Somasundaram, and R. Panneerselvam. 2009. Drought stress in plants: A review on morphological characteristics and pigments composition. Intl. J. Agr. Biol. 11:100-105.

Jia, X., C. Sun, G. Li, G. Li, and G. Chen. 2015. Effects of progressive drought stress on the physiology, antioxidative enzymes and secondary metabolites of Radix astragali. Acta Physiol. Plant. 37:262.

Kanehisa, M., S. Goto, Y. Sato, M. Kawashima, M. Furumichi, and M. Tanabe. 2014. Data, information, knowledge and principle: Back to metabolism in KEGG. Nucleic Acids Res. 42:199-205.

Kang, G., G. Li, G. Liu, W. Xu, X. Peng, C. Wang, Y. Zhu, and T. Guo. 2013. Exogenous salicylic acid enhances wheat drought tolerance by influence on the expression of genes related to ascorbate-glutathione cycle. Biol. Plant. 57:718-724.

Kebbas, S., S. Lutts, and F. Aid. 2015. Effect of drought stress on the photosynthesis of Acacia tortilis subsp. raddiana at the young seedling stage. Photosynthetica 53:288-298.
Kudo, M., S. Kidokoro, T. Yoshida, J. Mizoi, D. Todaka, A.R. Fernie, K. Shinozaki, and K. Yamaguchi-Shinozaki. 2017. Double overexpression of DREB and PIF transcription factors improves drought stress tolerance and cell elongation in transgenic plants. Plant Biotechnol. J. 15:458-471.

Li, S., X. Zhu, J. Zhang, G. Li, D. Su, and Y. Shan. 2012. Authentication of pure camellia oil by using near infrared spectroscopy and pattern recognition techniques. J. Food Sci. 77:C374-C380.

Liang, G., J. Bu, S. Zhang, J. Guo, G. Zhang, and X. Liu. 2019. Effects of drought stress on the photosynthetic physiological parameters of Populus $\times$ euramericana 'Neva'. J. For. Res. 30:409-416.

Maggio, A., S. Miyazaki, P. Veronese, T. Fujita, J.I. Ibeas, B. Damsz, M.L. Narasimhan, P.M. Hasegawa, R.J. Joly, and R.A. Bressan. 2002. Does proline accumulation play an active role in stressinduced growth reduction? Plant J. 31:699-712.

Mansour, M.M.F. 2013. Plasma membrane permeability as an indicator of salt tolerance in plants. Biol. Plant. 57:1-10.

Marcek, T., K.A. Hamow, B. Vegh, T. Janda, and E. Darko. 2019. Metabolic response to drought in six winter wheat genotypes. PLoS One 14:e0212411.

Mafakheri, A., A. Siosemardeh, B. Bahramnejad, P.C. Struik, and E. Sohrabi. 2010. Effect of drought stress on yield, proline and chlorophyll contents in three chickpea cultivars. Austral. J. Crop Sci. 4:580-585.

Marcińska, I., I. Czyczyło-Mysza, E. Skrzypek, M. Filek, S. Grzesiak, M.T. Grzesiak, F. Janowiak, T. Hura, M. Dziurka, K. Dziurka, A. Nowakowska, and S.A. Quarrie. 2013. Impact of osmotic stress on physiological and biochemical characteristics in drought-susceptible and drought-resistant wheat genotypes. Acta Physiol. Plant. 35:451461.

Miller, G., N. Suzuki, S. Ciftci-Yilmaz, and R. Mittler. 2010. Reactive oxygen species homeostasis and signalling during drought and salinity stresses. Plant Cell Environ. 33:453-467.

Nijs, I., R. Ferris, and H. Blum. 1997. Stomatal regulation in a changing climate: A field study using free air temperature increase (FATI) and free air $\mathrm{CO}_{2}$ enrichment (FACE). Plant Cell Environ. 20:1041-1050.

Salama, K.H.A. and M.M.F. Mansour. 2015. Choline priming-induced plasma membrane lipid alterations contributed to improved wheat salt tolerance. Acta Physiol. Plant. 37:170.

Todaka, D., Y. Zhao, T. Yoshida, M. Kudo, S. Kidokoro, J. Mizoi, K.S. Kodaira, Y. Takebayashi, M. Kojima, H. Sakakibara, K. Toyooka, M. Sato, A.R. Fernie, K. Shinozaki, and K. Yamaguchi-Shinozaki. 2017. Temporal and spatial changes in gene expression, metabolite accumulation and phytohormone content in rice seedlings grown under drought stress conditions. Plant J. 90:61-78.

Wang, Z., G. Li, H. Sun, L. Ma, Y. Guo, Z. Zhao, H. Gao, and L. Mei. 2018. Effects of drought stress on photosynthesis and photosynthetic electron transport chain in young apple tree leaves. Biol. Open 7:bio035279.

Yang, C., X. Liu, Z. Chen, Y. Lin, and S. Wang. 2016. Comparison of oil content and fatty acid profile of ten new Camellia oleifera cultivars. J. Lipids 2016:3982486.

Yang, D., J. Zhang, M. Li, and L. Shi. 2017. Metabolomics analysis reveals the salt-tolerant mechanism in Glycine soja. J. Plant Growth Regul. 36:460-471.

Zhang, J., D. Yang, M. Li, and L. Shi. 2016. Metabolic profiles reveal changes in wild and cultivated soybean seedling leaves under salt stress. PLoS One 11:e0159622.

Zhang, J., Y. Zhang, Y. Du, S. Chen, and H. Tang. 2011. Dynamic metabonomic responses of tobacco (Nicotiana tabacum) plants to salt stress. J. Proteome Res. 10:1904-1914.

Zhong, F., R. Wang, T. Li, P. Zhou, W. Liao, Y. Zhou, and R. Wang. 2015. Effects of soil moisture on major economic indexes of Camellia oleifera fruits. Nonwood For. Res. 33:32-37.

Zhuang, R. 2008. Camellia oleifera in China. 2nd ed. For. Press China, Beijing, China. 